\title{
Associação entre fatores socioeconômicos, comportamentais, saúde geral e condição da mucosa bucal em idosos
}

\author{
Association between socioeconomic factors, behavioral, \\ general health and oral mucosa status in elderly
}

Marisa Borges Oliveira ${ }^{1}$

Fernanda Ferreira Lopes ${ }^{1}$

Vandilson Pinheiro Rodrigues ${ }^{1}$

Claudia Maria Coelho Alves ${ }^{1}$

Fernando Neves Hugo ${ }^{2}$

${ }^{1}$ Programa de PósGraduação em Odontologia, Universidade Federal do Maranhão. Avenida dos Portugueses s $\mathrm{n}$, Bacanga. 65080-805 São Luís MA Brasil.marisaborges13@ hotmail.com

${ }^{2}$ Centro de Pesquisas em Odontologia Social, Universidade Federal do Rio Grande do Sul. Porto Alegre RS Brasil.

\begin{abstract}
This study aimed to investigate the association between socioeconomic and behavioral factors, general health, oral health, and plaque accumulation and oral mucosa condition outcomes in the elderly. This is a cross-sectional study conducted from 2004 to 2005 with 785 elderly dwellers of the city of Carlos Barbosa (RS), Brasil. We used questionnaires to collect socioeconomic, behavior and health status data. The physical examination of the oral structures comprised the Mucosal-Plaque Index proposed by Henriksen (MPS). A higher prevalence of moderate/severe plaque accumulation was observed in the elderly group, in less educated male elderly. The use of full upper dentures and income under one minimum wage were protective factors for moderate/severe plaque accumulation. Only the variables of the first block of the conceptual structure, male gender $(O R=2.13 ; 95 \%$ CI 1.26-3.61) and lower education $(O R=1.37 ; 95 \%$ CI 1.06-1.78) remained associated with an unacceptable MPS score. The findings suggest that, as per MPS, less educated male elderly are more likely to have poor oral health.
\end{abstract}

Key words Aging, Oral health, Oral mucosa, Socioeconomic factors
Resumo O objetivo deste estudo foi investigar a associação entre fatores socioeconômicos, comportamentais, saúde geral, saúde bucal e os desfechos de acúmulo de placa e condição de mucosa bucal em idosos. Este estudo transversal, conduzido entre 2004 e 2005, incluiu 785 idosos de Carlos Barbosa, RS. Questionários foram utilizados para coleta de dados socioeconômicos, comportamentais e estado de saúde. O exame físico das estruturas bucais e o Indice de Mucosa e de Placa de Henriksen (MPS) foram utilizados para categorizar os desfechos. Observou-se maior prevalência de acúmulo de placa moderado/grave entre os idosos mais velhos, do sexo masculino e com menor escolaridade. $O$ uso de prótese dental total superior e renda de até 1 salário mínimo foram fatores de proteção para acúmulo de placa moderado/grave. Apenas as variáveis do primeiro bloco da estrutura conceitual, sexo masculino $(O R=2,13 ; I C 95 \%=1,26-3,61)$ e menor escolaridade $(O R=1,37$; IC95\% $=1,06$ $1,78)$ permaneceram associados como risco à presença de escore inaceitável do MPS. Os achados sugerem que idosos do sexo masculino e com menor escolaridade têm maior chance de saúde bucal precária, conforme o MPS.

Palavras-chave Envelhecimento, Saúde bucal, Mucosa bucal, Fatores socioeconômicos 


\section{Introdução}

O envelhecimento é um processo fisiológico e heterogêneo, que pode predispor o indivíduo a patologias, como as lesões e infecções bucais, que frequentemente resultam da interação de diversos fatores como: uso de próteses dentais, microrganismos, nutrição, alterações hormonais e redução do fluxo salivar ${ }^{1-3}$. Variáveis socioeconômicas como a educação, renda, gênero ${ }^{4}$, a rede de apoio social e depressão ${ }^{5-7}$ também parecem estar associados a condição de saúde bucal dos idosos. Além disso, algumas doenças crônicas e neurodegenerativas podem comprometer as práticas em higiene bucal e dificultar o acesso aos serviços odontológicos, incrementando o impacto sobre os agravos em saúde bucal ${ }^{8-11}$.

Os agravos bucais mais frequentes nos idosos são a cárie dental, doença periodontal, perda dentária e edentulismo, associadas a doenças sistêmicas, medicamentos e uso de próteses dentais $^{12,13}$. O levantamento de saúde bucal realizado no Brasil no ano de 2010 revelou que a população de idosos apresentava os piores indicadores de saúde bucal, com alta prevalência de edentulismo, quando comparados aos demais grupos etários avaliados ${ }^{14}$. Estudos realizados em diferentes países também sugerem que as lesões da mucosa oral são comuns em idosos, estando associadas a fatores comportamentais e uso de medicamentos ${ }^{15-18}$.

Os Determinantes Sociais de Saúde (DSS) expressam a relação entre as condições de vida, trabalho, fatores sociais, econômicos, culturais, étnico, psicológicos e comportamentais de pessoas ou de grupos da população e sua condição de saúde ${ }^{19}$. Esse fato ressalta a importância do estudo destes fatores e sua potencial influência sobre a qualidade de vida das populações. Entretanto, no Brasil, estudos que investiguem a associação entre DDS e alterações de mucosa bucal em idosos ainda são escassos.

Com base no exposto, é necessária maior compreensão da influência dos determinantes sociais nas condições de saúde bucal da população idosa, para que se identifiquem fatores associados e ocorra um planejamento de ações de saúde bucal mais contextualizadas e resolutivas para esta população, visando melhorias na qualidade de vida. Nossa hipótese é que a pior condição de saúde bucal em idosos está relacionada a piores fatores socioeconômicos, comportamentais, saúde geral e psicológicos. Assim, o objetivo deste estudo foi investigar a associação entre esses fatores e a presença de alterações na mucosa e acúmulo placa bacteriana na cavidade bucal de idosos vivendo na comunidade.

\section{Metodologia}

O presente estudo transversal analítico foi realizado com uma amostra de idosos independentes com 60 anos ou mais, entre os anos de 2004 e 2005, na cidade de Carlos Barbosa, Rio Grande do Sul, Brasil. Do registro municipal de 2.167 idosos, 22 foram considerados inelegíveis por residirem em casa de repouso. Foi realizado o cálculo para amostras aleatórias simples, considerando o percentual da variável de interesse de $50 \%$, erro amostral de 3\% e nível de confiança a 99\% e uma taxa de perda de $11,5 \%$, resultando num tamanho amostral igual a 1106 indivíduos. Constatou-se na triagem inicial que 9 idosos haviam falecido, 1 havia se mudado para outro município e 113 não foram encontrados. Entre os 983 contatados, 13 estavam acamados, 1 pessoa estava hospitalizada e 97 recusaram-se participar do estudo. Assim, 872 pessoas participaram do estudo inicial, sendo que 785 completaram o protocolo analisado no presente estudo (Figura 1).

As entrevistas dos participantes ocorreram previamente aos exames bucais, realizados em consultórios odontológicos das Unidades Básicas de Saúde do Município. Este estudo apenas foi iniciado após a aprovação do Comitê de Ética em Pesquisa da Universidade Federal do Rio Grande do Sul. Todos os participantes foram informados sobre os objetivos e procedimentos do estudo e assinaram o termo de consentimento livre e esclarecido.

As variáveis socioeconômicas e demográficas foram: idade (em anos, para fins de análise categorizada em quartis), sexo, localização da residência (zona urbana ou rural), estado marital (casado, viúvo, outros), renda individual mensal (em salários mínimos, categorizada em $\leq 1$ salário mínimo ou > 1 salário mínimo), escolaridade (categorizada em $<4$ anos, 4 anos ou $>4$ anos de ensino formal completados). Informações sobre condições de saúde e comportamento em saúde incluíram a quantidade de medicamentos prescritos utilizados (não usava, usava apenas 1, usava 2 ou mais), comorbidades (possuía até 1 doença ou $\geq 2$ doenças, conforme relato de diagnóstico médico), uso de cigarros no momento da coleta de dados (sim ou não), sintomas de depressão de acordo com a Escala de Depressão Geriátrica (sem sintomas ou com sintomas de depressão), informações sobre condição protética superior e 
inferior (sem prótese, prótese parcial removível, prótese fixa, prótese fixa e parcial removível ou prótese total), fluxo salivar em repouso (em $\mathrm{ml} /$ min), escovação dental diária (mais de 2 vezes, 2 vezes, menos de 2 vezes).

Os exames clínicos foram realizados por dois examinadores experientes e previamente treinados. O coeficiente Kappa intra e inter examinadores, respectivamente 0,98 e 0,97 , foi verificado para o Índice MPS antes do estudo, indicando ótima concordância e replicabilidade. Os exames clínicos foram realizados com os indivíduos sentados em cadeira odontológica usando luz artificial. A inspeção intrabucal foi realizada com a ajuda de espelho bucal. As próteses foram removidas da boca, de modo que a inspeção de toda cavidade bucal do indivíduo fosse possível.

A presença e tipo de prótese dental foram registrados para maxila e mandíbula. Foi utilizado o Índice de Mucosa e de Placa de Henriksen (MPS), que fornece uma rápida avaliação geral da saúde bucal do indivíduo, observando o grau de inflamação da mucosa e da quantidade de placa bacteriana ao redor dos dentes e próteses ${ }^{20}$. A mucosa foi pontuada de acordo com o aspecto clínico usando a seguinte numeração: 1 - Aparência normal da mucosa da gengiva e palato; 2 - Ligeira inflamação, com áreas levemente vermelhas e/ou hipertrofia/hiperplasia da gengiva e/ ou áreas levemente vermelhas na mucosa do palato; 3 - Inflamação moderada, com áreas marcadamente vermelhas e hipertrofia/hiperplasia da gengiva, que sangra facilmente quando aplicada pressão, e/ou grandes áreas marcadamente vermelhas do palato ( $1 / 3$ ou mais), áreas marcadamente vermelhas e inflamadas na mucosa bucal e outras regiões fora do palato, ulceração causada por prótese, hiperplasias fibrosas vermelhas e inflamadas causadas por prótese; 4 - Inflamação grave, com vermelhidão severa e hipertrofia/hiperplasia da gengiva, sangramento gengival espontâneo, granulações marcadas no palato, áreas que facilmente se rompem, inflamadas e que sangram sob pressão de prótese dentária quando inserida na cavidade bucal. O índice de placa foi baseado em aspectos clínicos de acúmulo de biofilme na cavidade bucal (sobre próteses e dentes) utilizando os seguintes critérios: 1 - Sem placa claramente visível; 2 - Pequenas quantidades de placa visível; 3 - quantidades moderadas de placa visível; 4 - Quantidades abundantes de placa confluente. O escore MPS é constituído pela soma da pontuação dos escores de mucosa e da placa, variando de 2 a 8. O MPS é classificado como bom ou aceitável quando entre 2 e 4, como inaceitável 
quando entre 5 e 6, e como muito precário quando entre 7 e 8 . Neste estudo foi utilizada uma pontuação de 5 ou maior para definir inaceitável/ precário. Para efeito de calibragem neste estudo, foi utilizado um manual ilustrado contendo 25 fotografias a cores produzido no Setor de Gerontologia na Faculdade de Odontologia da Universidade de Oslo, Noruega ${ }^{20}$.

A coleta da saliva não estimulada seguiu os critérios propostos por Navazesh ${ }^{21}$. A coleta iniciou-se instruindo o participante para que a saliva fosse engolida, em sequência solicitou-se ao participante que deixasse o volume de saliva acumular na boca sem estimulação causada pelos movimentos orofaciais. Após 5 minutos, os participantes expectoravam em um tubo coletor graduado. O fluxo salivar em repouso foi expresso em $\mathrm{ml} / \mathrm{min}$ e os valores $<0,1 \mathrm{ml} / \mathrm{min}$ foram considerados hipossalivação ${ }^{22}$.

Para análise dos dados, um modelo teórico foi elaborado com base na estrutura conceitual proposta por Andersen e Davidson ${ }^{23}$. O Modelo utilizado no presente estudo foi constituído por quatro blocos. O primeiro bloco é mais distal em relação ao desfecho, sendo formado pela variável Exógena Idade (igual ou maior que 60 anos). O segundo bloco está relacionado aos determinantes primários, contendo as variáveis de características pessoais predisponentes (gênero, local de residência, estado civil, escolaridade) e recursos disponíveis (renda). O terceiro bloco, intermediário, em relação ao desfecho, relaciona-se ao comportamento de saúde bucal, com as seguintes variáveis: escovação dental e tabagismo. O quarto bloco é o mais proximal ao desfecho e refere-se às condições de saúde bucal, representadas pelas variáveis: estado de saúde geral (quantidade de medicamentos, comorbidades, GDS), e saúde bucal (condições protéticas, fluxo salivar). Os desfechos avaliados foram caracterizados pela presença de alterações na mucosa e placa bacteriana na cavidade bucal, por meio do índice MPS.

Foram analisados separadamente os desfechos MS, PS, e MPS, sendo que o MS e o PS foram categorizados em ausente ou leve (escores 1 e 2) e moderado ou severo (escores 3 e 4), e o escore MPS foi categorizado para MPS entre 1 e 4 (aceitável) ou MPS entre 5 e 8 (inaceitável/ péssimo). Para análise bivariada, o teste Quiquadrado e a medida Odds Ratio (OR) com respectivo intervalo de confiança a 95\% (IC95\%) foram utilizados para verificar a associação entre as variáveis de exposição, enquadradas em três grupos principais (socioeconômicas, comportamento de saúde e saúde bucal), e os desfechos
(MS, PS, MPS). Com o objetivo de reduzir o viés de confundimento, foi realizado o procedimento de análise multivariada através da regressão logística, utilizando a abordagem hierarquizada, na qual foram incluídas no modelo as variáveis que apresenta valor de $\mathrm{p}$ menor que 0,10 na análise bivariada, obtendo-se a medida OR ajustada. Os testes foram realizados através do Software SPSS (versão 18.0). O nível de significância estabelecido foi de $5 \%(\mathrm{p}<0,05)$.

\section{Resultados}

A idade média dos idosos foi de 68, $3 \pm 6,6$ anos, sendo a maioria do sexo feminino $(62,5 \%)$, casados $(71,3 \%)$ e não fumantes $(74,1 \%)$. A maior parte residia na zona rural (52,0\%), completou 4 anos de estudo $(40,4 \%)$ e possuía renda inferior ou igual a um salário mínimo (61,9\%).

A Tabela 1 expressa a distribuição percentual das variáveis referentes aos determinantes primários entre as categorias dos índices MS, PS ou MPS. Observou-se que as faixas etárias avaliadas apresentaram diferenças estatisticamente significantes apenas para o índice PS $(p=0,01)$, a maioria idosos com escore de PS ausente ou leve apresentava idade igual ou menor que 67 anos, enquanto que $54,6 \%$ dos idosos classificados na categoria moderado ou severo possuía idade igual ou superior a 68 anos. Constatou-se também diferenças estatisticamente significantes na distribuição da variável sexo para o índice PS ( $p$ $=0,001$ ), os homens representavam 48,3\% do escore moderado ou severo de PS, e apenas 25,6\% do escore ausente ou leve. A renda menor ou igual a um salário mínimo apresentou frequência estatisticamente mais elevada no escore moderado ou severo $(\mathrm{p}=0,01)$. Observou-se também um percentual maior de idosos com escolaridade menor que a $4^{\mathrm{a}}$ série no grupo de PS moderado ou severo (47,8\%; $\mathrm{p}=0,002)$. Para o índice MPS, constatou-se que um percentual estatisticamente maior ( $\mathrm{p}<0,05)$ de homens $(44,5 \%)$ e de idosos com escolaridade menor que a $4^{\text {a }}$ série $(47,6 \%)$ no grupo classificado como inaceitável. Não houve diferenças estatisticamente significantes na distribuição dos determinantes primários para os grupos de escore de MS.

A análise da frequência das variáveis referentes à saúde geral e bucal entre as categorias dos escores de MS, PS ou MPS está sumarizada na Tabela 2. Não houve diferenças estatisticamente significantes entre as condições de saúde avaliadas e o índice MS. Entretanto, observaram-se di- 


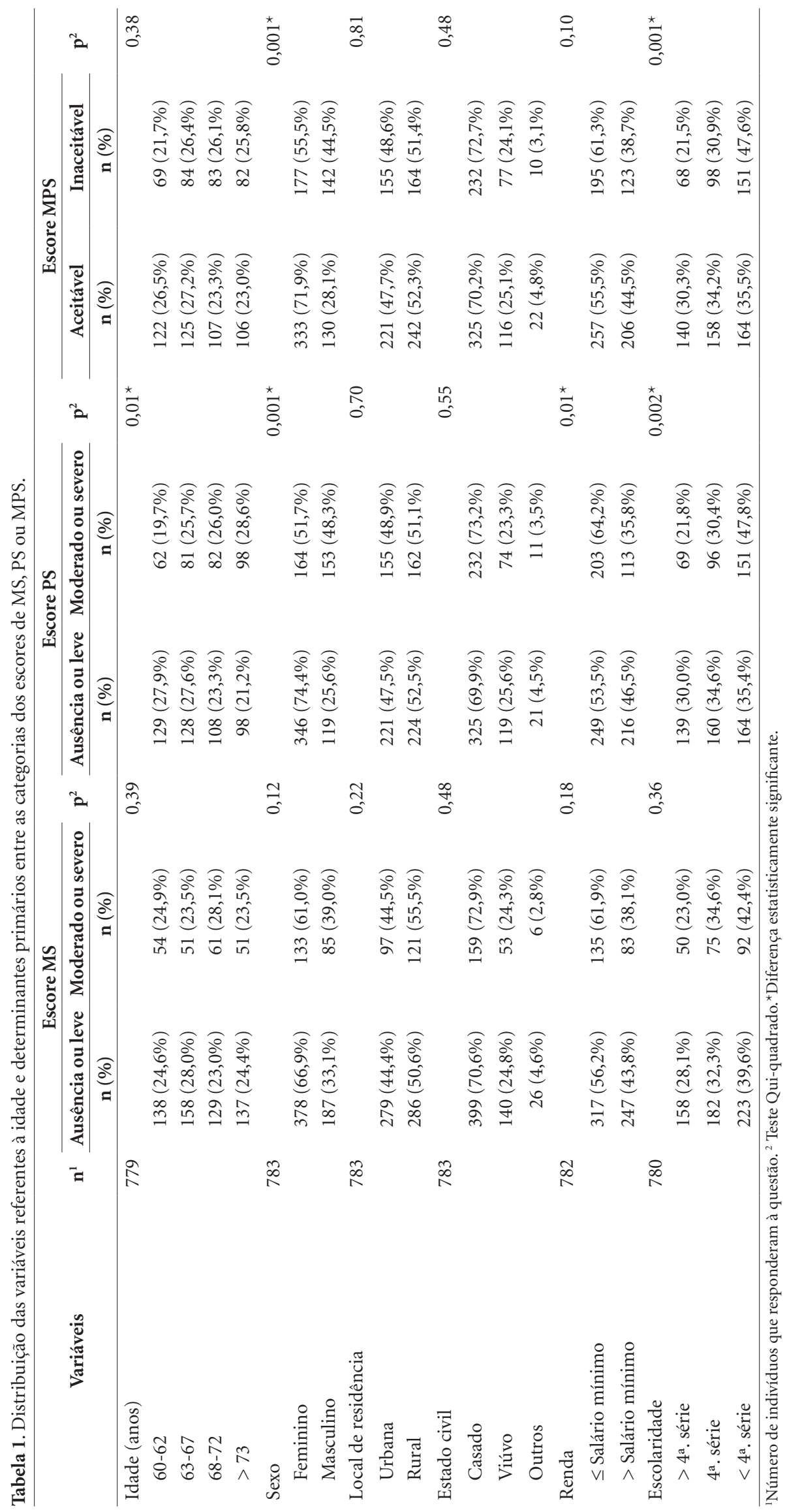


ferenças das categorias de escore PS e as variáveis escovação dental diária $(\mathrm{p}=0,01)$, tabagismo ( $\mathrm{p}$ $=0,01)$, prótese dental superior $(\mathrm{p}=0,03)$ e inferior $(\mathrm{p}=0,001)$. $\mathrm{O}$ grupo de escore PS moderado ou severo apresentou um maior percentual de idosos que relataram realizar até uma escovação dental diária $(33,2 \%)$, ser atual fumante $(33,3 \%)$, não usar prótese dental superior $(10,4 \%)$ e não usar prótese dental inferior (41,8\%), comparado ao grupo de escore PS ausente ou leve. $O$ índice MPS apresentou diferenças estatisticamente significantes na distribuição da variável escovação dental diária $(\mathrm{p}=0,03)$ e prótese dental inferior $(\mathrm{p}=$ inferior $)$, como maior percentual de idosos que relataram até uma escovação diária $(33,1 \%)$ e ausência de prótese dental inferior $(39,6 \%)$ no grupo de MPS inaceitável. As variáveis fluxo salivar, medicamentos, comorbidades e escala de depressão geriátrica não apresentaram diferenças estatisticamente significantes entre os escore de MS, PS e MPS.

As medidas de associação bruta e ajustada entre os determinantes primários e os desfechos estão representadas na Tabela 3. Após ajuste para potenciais confundidores, observou-se que chance de apresentar índice PS moderada/grave foi de 2,51 vezes maior em idosos do sexo masculino, 1,48 vezes maior entre os idosos de menor escolaridade, e a menor renda apresentou efeito protetor $(\mathrm{OR}$ ajustado $=0,52$; IC $95 \%=0,33-0,82)$. Para o índice MPS, as medidas ajustadas mostraram que os idosos do sexo masculino apresentaram maior risco de MPS inaceitável (OR ajustado $=2,13$; IC95\% = 1,26-3,61) e idosos com menor escolaridade também apresentaram maior risco de MPS inaceitável (OR ajustado = 1,37; IC95\% $=1,06-1,78$ ).

Além disso, também se estimou as medidas de associação entre as variáveis referentes às condições de saúde e desfechos (Tabela 4). Permaneceu associada após a regressão logística, apenas a categoria prótese total $(\mathrm{PT})$ superior e escore PS, sugerindo efeito protetor da presença da PT superior para a ocorrência de índice PS moderado ou severo $(\mathrm{OR}$ ajustado $=0,73$; IC95\% $=$ $0,55-0,97)$.

\section{Discussão}

Os principais achados do presente estudo sugerem que gravidade das alterações na mucosa bucal, mensurada através do índice MS, não apresentou associação com as variáveis incluídas nesta investigação. Por outro lado, constatou-se que apresentaram maior prevalência de placa moderada/grave os idosos mais velhos, do sexo masculino e com menor escolaridade. $\mathrm{O}$ uso de prótese dental total superior e possuir renda de até 1 salário mínimo foram fatores de proteção para placa moderada/grave. Apenas as variáveis do primeiro bloco da estrutura conceitual, sexo masculino e menor escolaridade, permaneceram associados à presença de escore inaceitável do MPS.

Estudos que abordam a associação entre determinantes sociais e saúde bucal são relevantes para indicar ações mais eficientes e com abrangência populacional. No presente estudo a presença de placa bacteriana na cavidade bucal dos idosos, mensurada através dos índices PS e MPS, estive relacionada a determinados fatores demográficos e socioeconômicos, como sexo, renda e escolaridade. No entanto, não foi observada associação significativa entre as variáveis idade, sexo, local de residência, estado civil e renda com o escore de mucosa (MS). Uma possível explicação para estes resultados pode estar na hipótese que as variáveis isoladamente não possuam poder de desenvolver alterações em mucosa, sendo necessário que ocorra a interação de diversos fatores locais, como microorganismos, fator hormonal, hipossalivação, hábitos como dormir com a prótese ${ }^{16,19,24}$.

Os achados não revelaram associação, após o ajustamento, entre a presença de placa e frequência de escovação dental diária nos idosos, no entanto, foi observado como mais frequente o MPS aceitável e PS ausente ou leve em idosos que realizam 3 ou mais escovações dentais por dia. Embora a prática de higiene bucal pareça ser menos comum em pessoas mais velhas, o risco que os idosos possuem de desenvolver doenças está mais associado ao efeito cumulativo do longo tempo de exposição aos fatores de risco que devido à idade em $\mathrm{si}^{25}$. Ressalta-se ainda, que embora não tenha havido associação, a presença de placa bacteriana na cavidade bucal resulta da negligência de higiene pessoal, independentemente da idade. Estudos prévios demonstram associação entre higiene bucal, nível de placa bacteriana e condições socioeconômicas ${ }^{26-28}$.

Neste trabalho, os idosos que possuíam prótese dental total superior e menor renda apresentaram-se como fatores de proteção para a presença de placa bacteriana (PS). Um estudo com idosos realizado na Noruega ${ }^{20}$ constatou que idosos usuários prótese apresentaram melhor indicadores de MPS que aqueles que possuíam os dentes naturais.

Com base nestes dados, podemos sugerir que possa ser mais fácil remover as próteses e higie- 


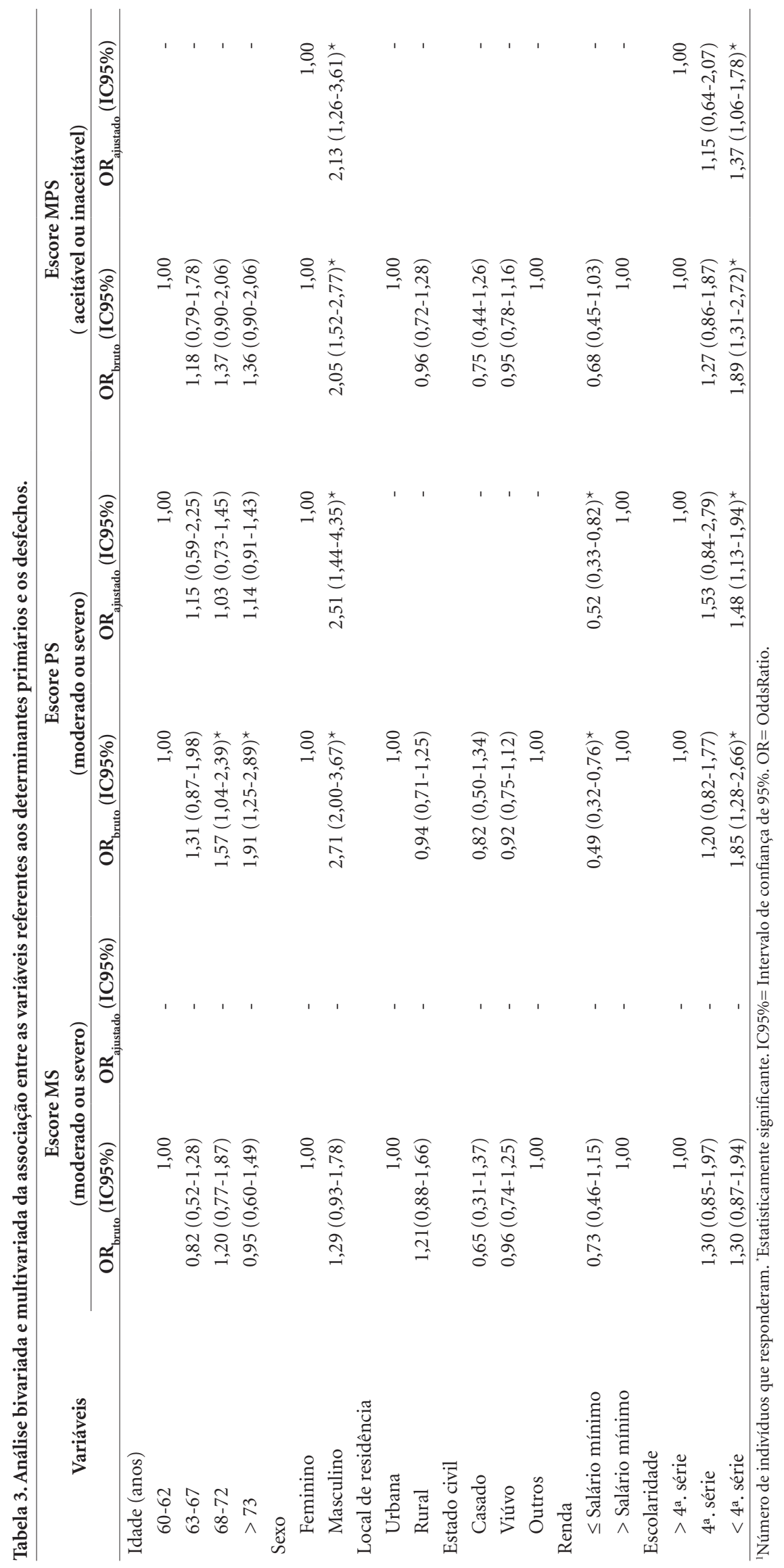




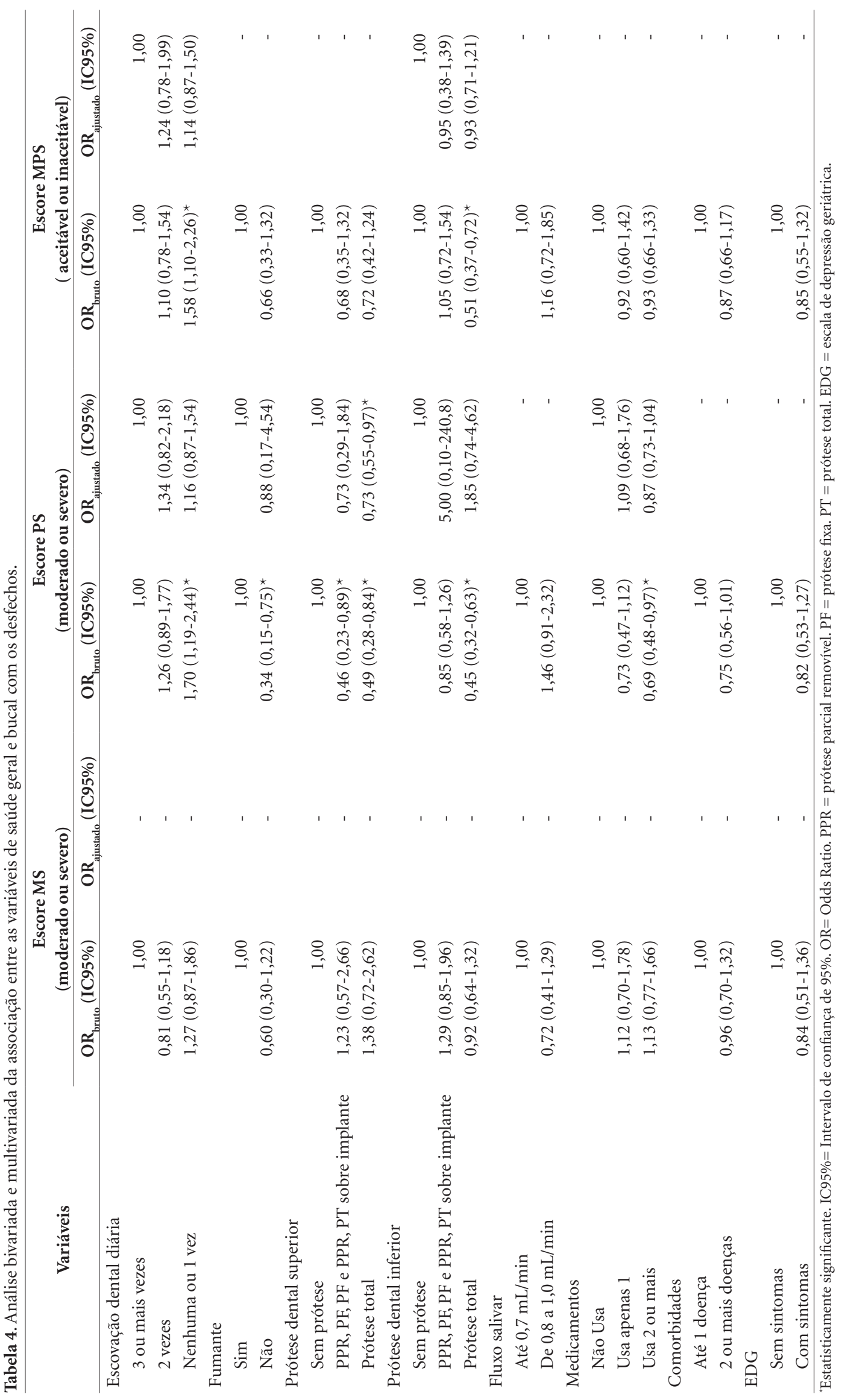


nizá-los eficientemente do que manter os dentes naturais limpos. $\mathrm{O}$ edentulismo, segundo alguns estudos, está fortemente associado a gênero ${ }^{29}$, baixa renda ${ }^{30} \mathrm{e}$ baixa escolaridade ${ }^{31}$.

Neste estudo, idosos do gênero masculino e possuir baixa escolaridade estiveram associados ao PS moderado/severo e MPS inaceitável. Resultados semelhantes foram obtidos por Heriksen ${ }^{20}$, que observaram que as mulheres apresentavam menores escores de MPS, sugerindo um reflexo de comportamentos diferentes entre os gêneros no auto-cuidado. Além do fator gênero, estudos anteriores demonstraram que os fatores que apresentavam maior associação com a negligência da higiene bucal em idosos são as condições socioeconômicas, escolaridade, educação precária em saúde e também o gênero ${ }^{27,28}$.

Dos fatores socioeconômicos, a escolaridade é considerada o mais básico, por moldar o potencial de trabalho e renda do indivíduo, além disso, influencia o acesso à informação, à promoção de saúde e prevenção de agravos ${ }^{32}$. A educação é uma categoria essencial na construção de uma sociedade, proporcionando aos cidadãos a proteção aos problemas sociais como baixa renda, desemprego, condições precárias de habitação, analfabetismo e de agravos na saúde, incluindo a saúde bucal ${ }^{33}$. No Brasil, os homens tendem a entrar no mercado de trabalho mais cedo que as mulheres, comprometendo o seu processo de escolaridade, com isso as mulheres apresentam nível educacional mais elevado ${ }^{34}$. A escolaridade, portanto, é um componente importante na determinação de hábitos e cuidados em saúde.

Existem vários métodos utilizados para verificar a presença de placa bacteriana em dentes e próteses, além de parâmetros de saúde periodontais, em indivíduos e populações. O índice MPS é um método simples, que verifica inflamação e presença de placa, de forma rápida e abrangente, sendo muito eficaz em estudos de populações como a de idosos, dispensando a utilização de outros índices na mensuração desses agravos. O caráter de avaliação mais global do índice MPS, assim como sua praticidade, foram fatores decisivos para a escolha da metodologia do presente estudo. Assim a utilização desse índice representa uma abordagem importante para fins de rastreamento ou classificação de problemas bucais em grupos ou populações como os idosos e associação com diversos fatores. Entretanto apesar de sua comprovada eficácia em estudos internacionais $^{20,35,36}$, ainda é pouco utilizado em estudos epidemiológicos em populações idosas ${ }^{34}$.

O estudo apresenta pontos fortes como a utilização do índice MPS e a boa representatividade da amostra da cidade de Carlos Barbosa no período da coleta de dados, além de alto poder estatístico com intervalos de confiança estreitos. A representatividade dos participantes foi verificada a partir dos dados sociais e demográficos da população de idosos de Carlos Brabosa. Não houve diferença significativa em relação à idade, sexo, estado civil e localização geográfica da residência entre os participantes do estudo e da população de pessoas com 60 anos ou mais de idade de Carlos Barbosa ${ }^{37}$.

Os achados desse estudo sugerem a associação de saúde bucal precária com fatores socioeconômicos entre idosos vivendo em comunidade. Idosos do sexo masculino e com menor escolaridade tiveram maior chance de apresentar acúmulo de placa bacteriana, por outro lado, aqueles que tinham menor renda e usavam prótese total estavam protegidos para a categoria mais grave de placa na cavidade bucal. As diferenças entre os indivíduos quanto às condições de saúde bucal, se dá pela posição social atrelada ao nível de escolaridade. A saúde bucal é um componente essencial para os cuidados de saúde para a população idosa, portanto políticas públicas contextualizadas com a realidade local são necessárias para garantir uma melhor qualidade de vida a esta população. 


\section{Colaboradores}

MB Oliveira trabalhou na concepção, desenho do estudo, análise dos dados e redação final, VP Rodrigues trabalhou na análise estatística dos dados, revisão e redação final, FF Lopes trabalhou no desenho do estudo, análise dos dados e redação final, CMC Alves trabalhou na concepção, análise dos dados e revisão final e FN Hugo trabalho na concepção do estudo, delineamento metodológico, análise dos dados e redação final.

\section{Agradecimentos}

À Fundação de Amparo à Pesquisa e ao Desenvolvimento Científico e Tecnológico do Maranhão - FAPEMA.

\section{Referências}

1. Affoo RH, Foley N, Garrick R, Siqueira WL, Martin RE. Meta-Analysis of Salivary Flow Rates in Young and Older Adults. J Am Geriatr Soc 2015; 63(10):2142-2151.

2. Lewis A, Wallace J, Deutsch A, King P. Improving the oral health of frail and functionally dependent elderly. Austral Dent J 2015; 60(S1):95-105.

3. Mariño R, Albala C, Sanchez H, Cea X, Fuentes A. Prevalence of diseases and conditions which impact on oral health and oral health self-care among older chilean. $J$ Aging Heal 2015; 27(1):3-16.

4. Yellowitz JA, Schneiderman MT. Elder's oral health crisis. J Evid Based Dent Pract 2014; 14(Supl.):191-200.

5. Campolina AG, Ciconelli RM. Qualidade de vida e medidas de utilidade: parâmetros clínicos para as tomadas de decisão em saúde. Rev Panam Salud Publ 2006; 19(2):128-136.

6. Cardoso EM, Parente RCP, Vettore MV, Rebelo MAB. Condição de saúde bucal em idosos residentes no município de Manaus, Amazonas: estimativas por sexo. Rev Bras Epidemiol 2011; 14(1):131-140.

7. Simões ACA, Carvalho DM. A realidade da saúde bucal do idoso no sudeste brasileiro. Cienc Saude Colt 2011; 16(6):2975-2982.

8. Soares EF, Novais TO, Freire MCM. Hábitos de higiene bucal e fatores relacionados em adultos de nível socioeconômico baixo. Rev Odontol UNESP 2009; 38(4):228234.

9. Andrade FB, Lebrao ML, Santos JLF, Duarte YAO. Relationship between oral health and frailty in community dwelling elderly individuals in Brazil. J Am Geriatr Soc 2013; 61(5):809-814.

10. Komulainen K, Ylöstalo P, Syrjälä AM, Ruoppi P, Knuuttila M, Sulkava R, Hartikainen S. Determinants for preventive oral health care need among community dwelling older people: a population-based study. Spec Care Dent 2014; 34(1):19-26.

11. Bots VantSpijker PC, Vanobbergen JN, Schols JM, Schaub RM, Bots CP, Baat C. Barriers of delivering oral health care to older people experienced by dentists: a systematic literature review. Comm Dent Oral Epidemiol 2014; 42(2):113-121.

12. Silva SRC, Valsecki Júnior A. Avaliação das condições de saúde dos idosos em um munucípio brasileiro. Rev Panam Salud Publ 2000; 8(4):268-271.

13. Petersen PE, Yamamoto T. Improving the oral health of older people: the approach of the WHO Global Oral Health Programme. Comm Dent Oral Epidemiol 2005; 33(2):81-92.

14. Peres MA, Barbato PR, Reis SCGB, Freitas CHSM, Antunes JLF. Perdas dentárias no Brasil: análise da Pesquisa Nacional de Saúde Bucal 2010. Rev Saud Publ 2013; 47(Supl. 3):78-89.

15. Griffin SO, Jones JA, Brunson D, Griffin PM, Bailey WD. Burden of oral disease among older adults and implications for public health priorities. Am J Publ Heal 2012; 102(3):411-418.

16. Jainkittivong A, Aneksuk V, Langlais RP. Oral mucosal conditions in elderly dental patients. Oral Dis 2002; 8(4):218-223.

17. Dundar N, Ilhan Kal B. Oral mucosal conditions and risk factors among elderly in a Turkish school of dentistry. Gerontol 2007; 53(3):165-172. 
18. Pentenero M, Broccoletti R, Carbone M, Conrotto D, Gandolfo $\mathrm{S}$. The prevalence of oral mucosal lesions in adults from the Turin area. Oral Dis 2008; 14(4):356366.

19. Lynge Pedersen AM, Nauntofte B, Smidt D, Torpet LA. Oral mucosal lesions in older people: relation to salivary secretion, systemic diseases and medications. Oral Dis 2015; 21(6):721-729.

20. Henriksen BM. Oral health among the elderly in Norway. A descriptive epidemiological study. Swed Dent J 2003; (Supl. 162):1-56.

21. Navazesh M. Methods for collecting saliva. Ann New York Acad Sci 1993; 694(1):72-77.

22. Bergdahl M, Bergdahl J. Low unstimulated salivary flow and subjective oral dryness: association with medication, anxiety, depression, and stress. J Dent Res 2000; 79(9):1652-1658.

23. Andersen RM, Davidson PL. Ethnicity, aging, and oral health outcomes: a conceptual framework. Adv Dent Res 1997; 11(2):203-209.

24. Cueto A, Martínez R, Niklander S, Deichler J, Barraza A, Esguep A. Prevalence of oral mucosal lesions in an elderly population in the city of Valparaiso, Chile. Gerodontol 2013; 30(3):201-206.

25. Petersen PE, Kandelman D, Arpin S, Ogawa H. Global oral health of older people-call for public health action. Comm Dent Healt 2010; 27(Supl. 2):257-267.

26. Evren BA, Uludamar A, Iseri U, Ozkan YK. The association between socioeconomic status, oral hygiene practice, denture stomatitis and oral status in elderly people living different residential homes. Arch Gerontol Geriatr 2011; 53(3):252-257.

27. Islas-Granillo H, Borges-Yañez SA, Medina-Solís CE, Casanova-Rosado AJ, Minaya-Sánchez M, Villalobos Rodelo JJ, Maupomé G. Socioeconomic, sociodemographic, and clinical variables associated with root caries in a group of persons age 60 years and older in Mexico. Geriatr Gerontol Int 2012; 12(2):271-276.

28. Kim HN, Ha TG, Kim, MJ, Junho EJ, Jeong, SH, Kim JB. Factors related to number of present teeth in Korean elderly adults aged 55-84 years. Int J Dent Hyg 2015; 14(2):151-158.

29. Susin C, Oppermann RV, Haugejorden O, Albandar JM. Tooth loss and associated risk indicators in an adult urban population from south Brazil. Acta Odontol Scand 2005; 63(2):85-93.
30. Dolan TA, Gilbert GH, Duncan RP, Foerster U. Risk indicators of edentulism, partial tooth loss and prosthetic status among black and white middle-aged and older adults. Comm Dent Oral Epidemiol 2001; 29(5):329340.

31. Cunha-Cruz J, Hujoel PP, Nadanovsky P. Secular trends in socio-economic disparities in edentulism: USA, 1972-2001. J Dent Res 2007; 86(2):131-136.

32. Fonesca FA, Jones KM, Mendes DC, dos Santos Neto PE, Ferreira RC, Pordeus IA, de Barros Lima Martins AME. The oral health of seniors in Brazil: addressing the consequences of a historic lack of public health dentistry in an unequal society. Gerodontol 2015; 32(1):18-27.

33. Adler NE, Newman K. Socioeconomic disparities in health: pathways and policies. Heal Aff 2002; 21(2):6076.

34. Portella FF, Rocha AW, Haddad DC, Fortes CB, Hugo FN, Padilha DM, Samuel SM. Oral hygiene caregivers educational programme improves oral health conditions in institutionalised independent and functional elderly. Gerodontol 2015; 32(1):28-34.

35. Henriksen BM, Ambjørnsen E, Laake K, Axéll TE. Oral hygiene and oral symptoms among the elderly in longterm care. Spec Care Dent 2004; 24(5):254-259.

36. Henriksen BM, Ambjørnsen E, Axéll TE. Evaluation of a mucosal-plaque index (MPS) designed to assess oral care in groups of elderly. Spec Care Dent 1999; 19(4):154-157.

37. Hugo FN, Hilgert JB, Sousa MLR, Cury JA. Oral status and its association with general quality of life in older independent-living south-Brazilians. Comm Dent Oral Epidemiol 2009; 37(3):231-240.

\footnotetext{
Artigo apresentado em 11/01/2016

Arovado em 15/11/2016

Versão final apresentada em 17/11/2016
} 